\title{
ODD-DIMENSIONAL COHOMOLOGY WITH FINITE COEFFICIENTS AND ROOTS OF UNITY
}

\author{
YURI G. ZARHIN
}

\begin{abstract}
We prove that the triviality of the Galois action on the suitably twisted odd-dimensional étale cohomology group with finite coefficients of an absolutely irreducible smooth projective variety implies the existence of certain primitive roots of unity in the field of definition of the variety. This text was inspired by an exercise in Serre's Lectures on the Mordell-Weil theorem.
\end{abstract}

\section{INTRODUCTION}

We recall some basic facts about cyclotomic characters. Let $K$ be a field, $\bar{K}$ its algebraic closure, $\mathrm{G}_{K}=\operatorname{Aut}(\bar{K} / K)$ the absolute Galois group of $K$. Let $n$ be a positive integer that is not divisible by $\operatorname{char}(K)$. We write $\mu_{n} \subset \bar{K}$ for the cyclic multiplicative group of $n$th roots of unity in $\bar{K}$. We write

$$
\bar{\chi}_{n}: \mathrm{G}_{K} \rightarrow \operatorname{Aut}\left(\mu_{n}\right)=(\mathbb{Z} / n \mathbb{Z})^{*}
$$

for the cyclotomic character that defines the Galois action on $n$th roots of unity. Clearly, $\mu_{n} \subset K$ if and only if

$$
\bar{\chi}_{n}(g)=1 \forall g \in \mathrm{G}_{K} .
$$

Recall that the order of $(\mathbb{Z} / n \mathbb{Z})^{*}$ is $\phi(n)$ where $\phi$ is the Euler function. This implies that

$$
\bar{\chi}_{n}^{\phi(n)}(g)=1 \forall g \in \mathrm{G}_{K} .
$$

Let $K\left(\mu_{n}\right) \subset \bar{K}$ be the $n$th cyclotomic extension of $K$. Then the degree $\left[K\left(\mu_{n}\right): K\right]$ of the abelian extension $K\left(\mu_{n}\right) / K$ coincides with the order of the finite commutative Galois group $\operatorname{Gal}\left(K\left(\mu_{n}\right) / K\right)$. By definition of $\bar{\chi}_{n}$, its kernel coincides with $\mathrm{G}_{K\left(\mu_{n}\right)}$ and $\bar{\chi}_{n}$ is the composition of the surjection

$$
\mathrm{G}_{K} \mapsto \mathrm{G}_{K} / \mathrm{G}_{K\left(\mu_{n}\right)}=\operatorname{Gal}\left(K\left(\mu_{n}\right) / K\right)
$$

and the embedding

$$
\operatorname{Gal}\left(K\left(\mu_{n}\right) / K\right)=\hookrightarrow(\mathbb{Z} / n \mathbb{Z})^{*},
$$

which we continue to denote by $\bar{\chi}_{n}$, slightly abusing notation.

Remark 1.1. Clearly, the exponent $\exp (n, K)$ of $\operatorname{Gal}\left(K\left(\mu_{n}\right) / K\right)$ divides the order of $\operatorname{Gal}\left(K\left(\mu_{n}\right) / K\right)$, which, in turn, divides $\phi(n)$. In addition, if $f$ is an integer then the character $\bar{\chi}_{n}^{f}$ is trivial if and only if $f$ is divisible by $\exp (n, K)$. In particular, the characters $\bar{\chi}_{n}^{\phi(n)}$ and $\bar{\chi}_{n}^{\exp (n, K)}$ are trivial. On the other hand, if the degree of the extension $K\left(\mu_{n}\right) / K$ is even then so is $\exp (n, K)$; this implies that if $f$ is an odd integer then the character $\bar{\chi}_{n}^{f}$ is nontrivial.

This work was partially supported by a grant from the Simons Foundation (\#246625 to Yuri Zarkhin). 
Remark 1.2. If $m$ is another positive integer that is relatively prime to $n$ and $\operatorname{char}(K)$, then the map

$$
\mu_{n} \times \mu_{m} \rightarrow \mu_{n m}, \quad\left(\gamma_{1}, \gamma_{2}\right) \mapsto \gamma_{1} \gamma_{2}
$$

is an isomorphism of groups (and even Galois modules). The natural map

$$
\phi_{n, m}: \mathbb{Z} / n m \mathbb{Z} \rightarrow \mathbb{Z} / n \mathbb{Z} \times \mathbb{Z} / m \mathbb{Z}, \quad c+n m \mathbb{Z} \mapsto(c+n \mathbb{Z}, c+m \mathbb{Z})
$$

is a ring homomorphism and the group homomorphism

$$
\bar{\chi}_{n m}: \mathrm{G}_{K} \rightarrow(\mathbb{Z} / n m \mathbb{Z})^{*}
$$

coincides with

$$
g \mapsto\left(\bar{\chi}_{n}(g), \bar{\chi}_{m}(g)\right) \in(\mathbb{Z} / n \mathbb{Z})^{*} \times(\mathbb{Z} / m \mathbb{Z})^{*} \stackrel{\phi_{n, m}^{-1}}{\longrightarrow}(\mathbb{Z} / n m \mathbb{Z})^{*} .
$$

If $A$ is an abelian variety over $K$ then we write $A[n]$ for the kernel of multiplication by $n$ in $A(\bar{K})$. It is well known that $A[n]$ is a finite Galois submodule of $A(\bar{K})$. If we forget about the Galois action then $A[n]$ is a free $\mathbb{Z} / n \mathbb{Z}$-module of rank $2 \operatorname{dim}(A)$.

The following assertion is stated without proof, as an exercise, in Serre's Lectures on the Mordell-Weil Theorem [7, Sect. 4.6, p. 55].

Theorem 1.3. If $\operatorname{dim}(A)>0$ and $A[n] \subset A(K)$ then $\mu_{n} \subset K$.

Proof. First, it suffices to check the case when $n=\ell^{r}$ is a power of a prime $\ell \neq$ $\operatorname{char}(K)$.

Second, if $A^{t}$ is the dual of $A$ then let us take a $K$-polarization $\lambda: A \rightarrow A^{t}$ of smallest possible degree. Then $\lambda$ is not divisible by $\ell$, i.e., $\operatorname{ker}(\lambda)$ does not contain the whole $A[\ell]$. Otherwise, divide $\lambda$ by $\ell$ to get a $K$-polarization of lower degree. Thus the image $\lambda\left(A\left[\ell^{r}\right]\right) \subset A^{t}\left[\ell^{r}\right]$ contains a point of exact order $\ell^{r}$, say $Q$. Otherwise,

$$
\lambda\left(A\left[\ell^{r}\right]\right) \subset A^{t}\left[\ell^{r-1}\right]
$$

and therefore $A[\ell]=\ell^{r-1} A\left[\ell^{r}\right]$ lies in the kernel of $\lambda$, which is not the case.

Since $A\left[\ell^{r}\right] \subset A[K]$ and $\lambda$ is defined over $K$, the image $\lambda\left(A\left[\ell^{r}\right]\right)$ lies in $A^{t}(K)$. In particular, $Q$ is a $K$-rational point on $A^{t}$.

Third, there is a nondegenerate Galois-equivariant Weil pairing [5]

$$
e_{n}: A\left[\ell^{r}\right] \times A^{t}\left[\ell^{r}\right] \rightarrow \mu_{\ell^{r}} .
$$

I claim that there is a point $P \in A\left[\ell^{r}\right]$ such that $e_{n}(P, Q)$ is a primitive $\ell^{r}$ th root of unity. Indeed, otherwise

$$
e_{n}\left(A\left[\ell^{r}\right], Q\right) \subset \mu_{\ell^{r-1}}
$$

so that the nonzero point $\ell^{r-1} Q$ is orthogonal to the whole $A\left[\ell^{r}\right]$ with respect to $e_{n}$, which contradicts the nondegeneracy of $e_{n}$.

Thus, $\gamma:=e_{n}(P, Q)$ is a primitive $\ell^{r}$ th root of unity that lies in $K$, because both $P$ and $Q$ are $K$-points. Since $\mu_{\ell^{r}}$ is generated by $\gamma, \mu_{\ell^{r}} \subset K$.

The aim of this paper is to a prove a variant of Serre's exercise that deals with the Galois action on the twisted odd-dimensional étale cohomogy group with finite coefficients of a smooth projective variety (see Theorem 1.6 below). Our proof is based on the Hard Lefschetz Theorem [2] and the unimodularity of Poincaré duality [10. 
1.4. If $\Lambda$ is a commutative ring with 1 and without zero divisors and $M$ is a $\Lambda$ module, then we write $M_{\text {tors }}$ for its torsion submodule and $M /$ tors for the quotient $M / M_{\text {tors }}$. Usually, we will use this notation when $\Lambda$ is the ring $\mathbb{Z}_{\ell}$ of $\ell$-adic integers.

If $\ell$ is a prime different from $\operatorname{char}(K)$ then we write $\mathbb{Z}_{\ell}(1)$ for the projective limit of the cyclic Galois modules $\mu_{\ell^{r}}$ with $\ell$ th power as transition map. It is known that $\mathbb{Z}_{\ell}(1)$ is a free $\mathbb{Z}_{\ell}$-module of rank 1 with natural continuous action of $G_{K}$ defined by the cyclotomic character

$$
\chi_{\ell}: \mathrm{G}_{K} \rightarrow \operatorname{Aut}_{\mathbb{Z}_{\ell}}\left(\mathbb{Z}_{\ell}(1)\right)=\mathbb{Z}_{\ell}^{*} .
$$

There are canonical isomorphisms

$$
\mathbb{Z}_{\ell} / \ell^{r} \mathbb{Z}_{\ell}=\mathbb{Z} / \ell^{r} \mathbb{Z}, \mathbb{Z}_{\ell}(1) / \ell^{r} \mathbb{Z}_{\ell}(1)=\mu_{\ell^{r}}
$$

in addition

$$
\chi_{\ell} \bmod \ell^{r}=\bar{\chi}_{\ell^{r}}
$$

for all positive integers $r$.

We write $\mathbb{Q}_{\ell}(1)$ for the one-dimensional $\mathbb{Q}_{\ell}$-vector space

$$
\mathbb{Q}_{\ell}(1)=\mathbb{Z}_{\ell}(1) \otimes_{\mathbb{Z}_{\ell}} \mathbb{Q}_{\ell}
$$

provided with the natural Galois action that is defined by the character $\chi_{\ell}$. For each integer $a$ we will need the $a$ th tensor power $\mathbb{Q}_{\ell}(a):=\mathbb{Q}_{\ell}(1)^{\otimes a}$, which is a one-dimensional $\mathbb{Q}_{\ell}$-vector space provided with the Galois action that is defined by the character $\chi_{\ell}^{a}$.

Let $X$ be an absolutely irreducible smooth projective variety over $K$ of positive dimension $d=\operatorname{dim}(X)$. We write $\bar{X}$ for the irreducible smooth projective $d$-dimensional variety $X \times_{K} \bar{K}$ over $\bar{K}$. Let $\ell$ be a prime $\neq \operatorname{char}(K)$ and $a$ an integer. If $i \leq 2 d$ is a nonnegative integer then we write $H^{i}\left(\bar{X}, \mathbb{Z}_{\ell}(a)\right)$ for the corresponding twisted $i$ th étale $\ell$-adic cohomology group. Recall that all the étale cohomology groups $H^{i}\left(\bar{X}, \mu_{n}^{\otimes a}\right)$ are finite $\mathbb{Z} / n \mathbb{Z}$-modules and that the $\mathbb{Z}_{\ell}$-modules $H^{i}\left(\bar{X}, \mathbb{Z}_{\ell}(a)\right)$ are finitely generated. In particular, each $H^{i}\left(\bar{X}, \mathbb{Z}_{\ell}(a)\right) /$ tors is a free

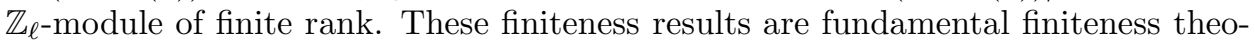

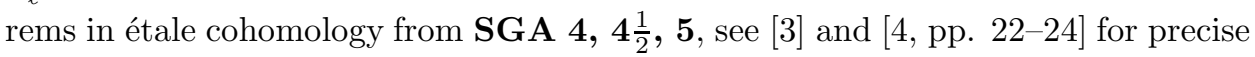
references. All these groups are provided with the natural linear continuous actions of $\mathrm{G}_{K}$. We also consider the corresponding finite-dimensional $\mathbb{Q}_{\ell}$-vector spaces $^{- \text {to }}$

$$
H^{i}\left(\bar{X}, \mathbb{Q}_{\ell}(a)\right)=H^{i}\left(\bar{X}, \mathbb{Z}_{\ell}(a)\right) \otimes_{\mathbb{Z}_{\ell}} \mathbb{Q}_{\ell} .
$$

The Galois action on $H^{i}\left(\bar{X}, \mathbb{Z}_{\ell}(a)\right)$ extends by $\mathbb{Q}_{\ell}$-linearity to $H^{i}\left(\bar{X}, \mathbb{Q}_{\ell}(a)\right)$. There are natural isomorphisms of $\mathrm{G}_{K}$-modules

$$
H^{i}\left(\bar{X}, \mathbb{Q}_{\ell}(a+b)\right)=H^{i}\left(\bar{X}, \mathbb{Q}_{\ell}(a)\right) \otimes_{\mathbb{Q}_{\ell}} \mathbb{Q}_{\ell}(b)
$$

for all integers $a$ and $b$.

Remark 1.5. If a positive integer $m$ is relatively prime to $n$ and $\operatorname{char}(K)$, then the splitting $\mu_{n m}=\mu_{n} \times \mu_{m}$ induces the splitting of Galois modules

$$
H^{i}\left(\bar{X}, \mu_{n m}{ }^{\otimes a}\right)=H^{i}\left(\bar{X}, \mu_{n}{ }^{\otimes a}\right) \oplus H^{i}\left(\bar{X}, \mu_{m}{ }^{\otimes a}\right) .
$$

The $\mathbb{Q}_{\ell^{-}}$dimension of $H^{i}\left(\bar{X}, \mathbb{Q}_{\ell}(a)\right)$ is denoted by $\mathbf{b}_{i}(\bar{X})$ and called the $i$ th Betti number of $\bar{X}$ : it does not depend on a choice of $(a$ and $) \ell$. In characteristic zero it follows from the comparison theorem between classical and étale cohomology [6]. In finite characteristic the independence follows from results of Deligne [1. It is also known that $\mathbf{b}_{i}(\bar{X})=0$ if $i>2 d \underline{4}, 3$. 
Our main result is the following statement.

Theorem 1.6. Let $i$ be a nonnegative integer.

(i) Suppose that $i \leq d-1$ and $\mathbf{b}_{2 i+1}(\bar{X}) \neq 0$. If the Galois action on $H^{2 i+1}\left(\bar{X}, \mu_{n}{ }^{\otimes i}\right)$ is trivial then $\mu_{n} \subset K$.

(ii) Suppose that $1 \leq i \leq d$ and $\mathbf{b}_{2 i-1}(\bar{X}) \neq 0$. If the Galois action on $H^{2 i-1}\left(\bar{X}, \mu_{n}{ }^{\otimes i}\right)$ is trivial then $\mu_{n} \subset K$.

Example 1.7. Let us take $i=1$. Then Kummer theory tells us that

$$
H^{2 i-1}\left(\bar{X}, \mu_{n}^{\otimes i}\right)=H^{1}\left(\bar{X}, \mu_{n}\right)=\operatorname{Pic}(\bar{X})[n]
$$

is the kernel of multiplication by $n$ in the Picard group $\operatorname{Pic}(\bar{X})$ of $\bar{X}$. On the other hand if $B$ is an abelian variety over $K$ that is the Picard variety of $X[5$ then $\operatorname{dim}(B)=\mathbf{b}_{1}(\bar{X})$ and $B[n]$ is a Galois submodule of $H^{1}\left(\bar{X}, \mu_{n}\right)$. If we know that the Galois action on $H^{1}\left(\bar{X}, \mu_{n}\right)$ is trivial then the same is true for its submodule $B[n]$. Now if $\mathbf{b}_{1}(\bar{X}) \neq 0$ then $B \neq\{0\}$ and Theorem 1.3 applied to $B$ implies that $\mu_{n} \subset K$

Theorem 1.6 may be viewed as a special case (when $a=\frac{j \pm 1}{2}$ ) of the following statement.

Theorem 1.8. Let $j$ be a nonnegative integer and $\mathbf{b}_{j}(\bar{X}) \neq 0$. Let a be an integer. Assume that the Galois action on $H^{j}\left(\bar{X}, \mu_{n}{ }^{\otimes a}\right)$ is trivial. Then

$$
\bar{\chi}_{n}^{2 a-j}(g)=1 \forall g \in G=\mathrm{G}_{K} .
$$

If, in addition, $2 a-j$ is relatively prime to $\phi(n)$ then $\mu_{n} \subset K$.

Corollary 1.9 (Corollary to Theorem 1.8). Let $K$ be a field, $n$ a positive integer prime to char $(K)$. Suppose that $K$ does not contain a primitive nth root of unity. Suppose that $j$ is an odd positive integer. Let a be an integer such that $2 a-j$ is relatively prime to $\phi(n)$. Then for each absolutely irreducible smooth projective variety $X$ over $K$ with $\mathbf{b}_{j}(\bar{X}) \neq 0$ the Galois group $\mathrm{G}_{K}$ acts nontrivially on $H^{j}\left(\bar{X}, \mu_{n}^{\otimes a}\right)$

The next assertion covers (in particular) the case of quadratic $\bar{\chi}_{n}$ (e.g., when $K$ is the maximal real subfield $\mathbb{Q}\left(\mu_{n}\right)^{+}$of the $n$th cyclotomic field $\mathbb{Q}\left(\mu_{n}\right)$ of $\mathbb{Q}$.)

Theorem 1.10. Let $K$ be a field, $n$ a positive integer prime to char $(K)$. Suppose that the degree $\left[K\left(\mu_{n}\right): K\right]$ is even. (E.g., $K\left(\mu_{n}\right) / K$ is a quadratic extension.) Then for each positive odd integer $j$, each integer a and every absolutely irreducible smooth projective variety $X$ over $K$ with $\mathbf{b}_{j}(\bar{X}) \neq 0$ the Galois group $\mathrm{G}_{K}$ acts nontrivially on $H^{j}\left(\bar{X}, \mu_{n}^{\otimes a}\right)$.

Remark 1.11. The special case of Theorem 1.10 when $\bar{\chi}_{n}$ is a quadratic character follows directly from Theorem [1.6, because in this case the Galois module $H^{j}\left(\bar{X}, \mu_{n}^{\otimes a}\right)$ is isomorphic either to $H^{j}\left(\bar{X}, \mu_{n}^{\otimes[(j+1) / 2]}\right)$ or to $H^{j}\left(\bar{X}, \mu_{n}^{\otimes[(j-1) / 2]}\right)$.

The paper is organized as follows. Section 2 contains auxiliary results about pairings between finitely generated modules over discrete valuation rings. We use them in Section 3, in order to prove Theorems 1.8, 1.6] and 1.10.

Acknowledgements. This work is a follow up of [8, 9, 10]. I am deeply grateful to Alexey Parshin for helpful comments. My thanks also go to Peter Schneider, Alice Silverberg, Adebisi Agboola, Alexei Skorobogatov and Nick Katz for their interest in this paper (and/or the previous ones). My very special thanks go to the referee, whose numerous suggestions and comments significantly improved the 
exposition. The final version of this paper was prepared during my stay at the Max-Planck-Institut für Mathematik (Bonn), whose hospitality and support are gratefully acknowledged.

\section{LineAr ALGeBra}

This section contains auxiliary results that will be used in the next section in order to prove main results of the paper.

2.1. Let $E$ be a discrete valuation field, $\Lambda \subset E$ the corresponding discrete valuation ring with maximal ideal $\mathfrak{m}$. Let $\pi \in \mathfrak{m}$ be an uniformizer, i.e., $\mathfrak{m}=\pi \Lambda$.

If $U$ is a finitely generated $\Lambda$-module then we write $U_{E}$ for the corresponding finite-dimensional $E$-vector space $U \otimes_{\Lambda} E$. The kernel of the homomorphism of $\Lambda$-modules

$$
\otimes 1: U \rightarrow U \otimes_{\Lambda} E=U_{E}, x \mapsto x \otimes 1
$$

coincides with $U_{\text {tors }}$ while the image

$$
\tilde{U}:=\otimes 1(U) \subset U_{E}
$$

is a $\Lambda$-lattice in $V_{E}$ of maximal rank $\operatorname{dim}_{E}\left(U_{E}\right)$.

Let $G$ be a group and

$$
\chi: G \rightarrow \Lambda^{*} \subset E^{*}
$$

is a homomorphism of $G$ to the group $\Lambda^{*}$ of invertible elements of $\Lambda$. If $H$ is a nonzero finite-dimensional vector space over $E$ and

$$
\rho: G \rightarrow \operatorname{Aut}_{E}(H)
$$

is a $E$-linear representation of $G$ in $H$ then $H$ becomes a module over the group algebra $E[G]$ of $G$ over $E$. Then

$$
\rho \otimes \chi: G \rightarrow \operatorname{Aut}_{E}(H), \rho \otimes \chi(g)=\chi(g) \rho(g) \forall g \in G
$$

is also a linear representation of $G$ in $H$. We denote the corresponding $E[G]$-module by $H(\chi)$ and call it the twist of $H$ by $\chi$. Notice that $H$ and $H(\chi)$ coincide as $E$ vector spaces. It is also clear that if $T$ is a $\Lambda$-lattice in $H$ then it is $G$-stable in $H(\chi)$ if and only if it is $G$-stable in the $E[G]$-module $H$. On the other hand, let $L$ be a one-dimensional $E$-vector space provided with a structure of $G$-module defined by

$$
g z:=\chi(g) z \forall g \in G, z \in L .
$$

Then the $G$-modules $H(\chi)$ and $H \otimes_{E} L$ are isomorphic noncanonically.

Lemma 2.2. Suppose that $H_{1}$ and $H_{2}$ are nonzero finite-dimensional E-vector spaces and

$$
\rho_{1}: G \rightarrow \operatorname{Aut}_{E}\left(H_{1}\right), \rho_{2}: G \rightarrow \operatorname{Aut}_{E}\left(H_{2}\right)
$$

are isomorphic E-linear representations of $G$. Suppose that $T_{1}$ is a $G$-stable $\Lambda$ lattice in $H_{1}$ of rank $\operatorname{dim}_{E}\left(H_{1}\right)$ and $T_{2}$ is a $G$-stable $\Lambda$-lattice in $H_{2}$ of rank $\operatorname{dim}_{E}\left(H_{2}\right)$. Then there is an isomorphism of $E[G]$-modules $u: H_{1} \rightarrow H_{2}$ such that

$$
u\left(T_{1}\right) \subset T_{2}, u\left(T_{1}\right) \not \subset \pi \cdot T_{2} .
$$


Proof. Clearly,

$$
H_{2}=\bigcup_{j=1}^{\infty} \pi^{-j} \cdot T_{2}, \bigcap_{j=1}^{\infty} \pi^{j} \cdot T_{2}=\{0\} .
$$

Let $u_{0}: H_{1} \cong H_{2}$ be an isomorphism of $E[G]$-modules. Since $H_{1}$ is a finitely generated $\Lambda$-module, there exists an integer $j$ such that $\pi^{-j} \cdot u_{0}\left(T_{1}\right) \subset T_{2}$. Let us take the smallest $j$ that enjoys this property and put $u=\pi^{-j} u_{0}$.

Theorem 2.3. Suppose that $U$ and $V$ are finitely generated $\Lambda$-modules provided with group homomorphisms

$$
G \rightarrow \operatorname{Aut}_{\Lambda}(U), G \rightarrow \operatorname{Aut}_{\Lambda}(V) .
$$

Let us assume that $U /$ tors $\neq\{0\}$, i.e., rank of $U$ is positive.

Suppose that we are given a $\Lambda$-bilinear pairing

$$
e: U \times V \rightarrow \Lambda
$$

that enjoys the following properties.

(i)

$$
e(g x, g y)=\chi(g) \cdot e(x, y) \forall g \in G ; x \in U, y \in V .
$$

(ii) The $\Lambda$-bilinear pairing

$$
U / \text { tors } \times V / \text { tors } \rightarrow \Lambda
$$

induced by $e$ is perfect (unimodular).

(iii) The $E[G]$-modules $U_{E}$ and $V_{E}$ are isomorphic.

Let $r$ be a positive integer such that the induced $G$-action on $U / \pi^{r} U$ is trivial, i.e.,

Then

$$
x-g x \in \pi^{r} U \forall g \in G, x \in U .
$$

$$
\chi(g) \bmod \pi^{r} \Lambda=1 \in \Lambda / \pi^{r} \Lambda \forall g \in G .
$$

Proof. Clearly,

$$
e\left(U_{\text {tors }}, V\right)=\{0\}=e\left(U, V_{\text {tors }}\right) .
$$

Also $U_{\text {tors }}$ is a $G$-submodule of $U$ and $V_{\text {tors }}$ is a $G$-submodule of $V$. Moreover, the $G$-module $[U /$ tors $] / \pi^{r}[U /$ tors $]$ is isomorphic to a quotient of the $G$-module $U / \pi^{r} U$. In particular, the $G$-action on $[U /$ tors $] /\left[\pi^{r} U /\right.$ tors $]$ is also trivial. In the notation of Sect. 2.1 the natural homomorphisms

$U /$ tors $=U / U_{\text {tors }} \rightarrow \tilde{U}, x+U_{\text {tors }} \mapsto x \otimes 1, V /$ tors $=V / V_{\text {tors }} \rightarrow \tilde{V}, x+V_{\text {tors }} \mapsto x \otimes 1$ are $G$-equivariant isomorphisms of free $\Lambda$-modules of finite rank

$$
U / \text { tors } \cong \tilde{U}, V / \text { tors } \cong \tilde{V}
$$

where $\tilde{U}$ and $\tilde{V}$ are $G$-stable lattices of maximal rank in $U_{E}$ and $V_{E}$ respectively. This implies that the $G$-action on $\tilde{U} / \pi^{r} \tilde{U}$ and $e$ induces a $\Lambda$-bilinear perfect pairing

$$
\tilde{e}: \tilde{U} \times \tilde{V} \rightarrow \Lambda
$$

such that

$$
\tilde{e}(g x, g y)=\chi(g) \cdot \tilde{e}(x, y) \forall g \in G ; x \in \tilde{U}, y \in \tilde{V} .
$$

Applying Lemma 2.2 to the isomorphic $E[G]$-modules $U_{E}$ and $V_{E}$, we obtain a "nicer" isomorphism of $E[G]$-modules $u: U_{E} \cong V_{E}$ such that

$$
u\left(T_{1}\right) \subset T_{2}, u\left(T_{1}\right) \not \subset \pi T_{2} .
$$


Let us pick $x_{0} \in T_{1}$ with $y:=u\left(x_{0}\right) \notin \pi T_{2}$. Since $x_{0} \bmod \pi^{r} T_{1} \in T_{1} / \pi^{r} T_{1}$ is $G$-invariant, its image

$$
u(x) \bmod \pi^{r} T_{2}=y \bmod \pi^{r} T_{2} \in T_{2} / \pi^{r} T_{2}
$$

is also $G$-invariant. Since $y$ is not divisible in $T_{2}$, the $\Lambda$-submodule $\Lambda \cdot y$ is a direct summand of $T_{2}$. Since the pairing $\tilde{e}$ between $T_{1}$ and $T_{2}$ is perfect, there is $x \in T_{1}$ with $e(x, y)=1$. This implies that

$$
\chi(g)=\chi(g) \cdot 1=\chi(g) \cdot \tilde{e}(x, y)=\tilde{e}(g x, g y),
$$

i.e.,

$$
\chi(g)=\tilde{e}(g x, g y) \forall g \in G .
$$

On the other hand, since

$$
x-g x \in \pi^{r} T_{1}, y-g y \in \pi^{r} T_{2},
$$

we have

$$
\tilde{e}(g x, g y)-\tilde{e}(x, y) \in \pi^{r} \Lambda \forall g \in G .
$$

This means that

$$
\chi(g)-1=\tilde{e}(g x, g y)-\tilde{e}(x, y) \in \pi^{r} \Lambda \forall g \in G
$$

and we are done.

The next statement is a useful variant of Theorem 2.3 that deals with twisted representations.

Theorem 2.4. Suppose that $U$ and $V$ are finitely generated $\Lambda$-modules provided with group homomorphisms

$$
G \rightarrow \operatorname{Aut}_{\Lambda}(U), G \rightarrow \operatorname{Aut}_{\Lambda}(V) .
$$

Assume that $U /$ tors $\neq\{0\}$, i.e. the rank of $U$ is positive.

Suppose that we have a $\Lambda$-bilinear pairing

$$
e: U \times V \rightarrow \Lambda
$$

that enjoys the following properties.

(i)

$$
e(g x, g y)=e(x, y) \forall g \in G ; x \in U, y \in V .
$$

(ii) The $\Lambda$-bilinear pairing

$$
U / \text { tors } \times V / \text { tors } \rightarrow \Lambda
$$

induced by $e$ is perfect (unimodular).

(iii) The $E[G]$-modules $U_{E}$ and $V_{E}(\chi)$ are isomorphic.

Let $r$ be a positive integer such that the induced $G$-action on $U / \pi^{r} U$ is trivial, i.e.

$$
x-g x \in \pi^{r} U \forall g \in G, x \in U .
$$

Then

$$
\chi(g) \bmod \pi^{r} \Lambda=1 \in \Lambda / \pi^{r} \Lambda \forall g \in G .
$$


Proof. Let

$$
\rho_{U}: G \rightarrow \operatorname{Aut}_{\Lambda}(U), \rho_{V}: G \rightarrow \operatorname{Aut}_{\Lambda}(V)
$$

be the structure homomorphisms that define the actions of $G$ on $U$ and $V$ respectively. In this notation,

$$
e\left(\rho_{U}(g) x, \rho_{V}(g) y\right)=e(x, y) \forall g \in G ; x \in U, y \in V .
$$

Let us twist $\rho_{V}$ by considering the group homomorphism

$$
\rho_{V(\chi)}: G \rightarrow \operatorname{Aut}_{\Lambda}(V), g \mapsto \chi(g) \rho(g) .
$$

We denote the resulting $G$-module by $V(\chi)$ and call it the twist of $V$ by $\chi$. Notice that $V$ coincides with $V(\chi)$ as $\Lambda$-module. On the other hand, the $E[G]$-module $V(\chi)_{E}$ is canonically isomorphic to $V_{E}(\chi)$. The pairing $e$ defines the $\Lambda$-bilinear pairing

$$
e_{\chi}: U \times V(\chi) \rightarrow \Lambda, e_{\chi}(x, y):=e(x, y) \forall x \in U, y \in V=V(\chi)
$$

of $G$-modules $U$ and $V(\chi)$, which satisfies

$$
\begin{gathered}
e_{\chi}\left(\rho_{U}(g) x, \rho_{V(\chi)}(g) y\right)=e\left(\rho_{U}(g) x, \chi(g) \rho_{V}(g) y\right)=\chi(g) e\left(\rho_{U}(g) x, \rho_{V}(g) y\right)= \\
\chi(g) e(x, y)=\chi(g) e_{\chi}(x, y) \forall g \in G ; x \in U, y \in V(\chi) .
\end{gathered}
$$

This implies that

$$
e_{\chi}\left(\rho_{U}(g) x, \rho_{V(\chi)}(g) y\right)=\chi(g) e_{\chi}(x, y) \forall g \in G ; x \in U, y \in V(\chi) .
$$

Now the result follows from Theorem 2.3 applied to $U, V(\chi)$ and $e_{\chi}$.

\section{Proofs of MAin Results}

Let $\ell$ be a prime different from $\operatorname{char}(K)$ and $r$ a positive integer. Let us put

$$
E=\mathbb{Q}_{\ell}, \Lambda=\mathbb{Z}_{\ell}, \pi=\ell, G=\mathrm{G}_{K} .
$$

We keep the notation and assumptions of Sect. 1.4, Recall that $d=\operatorname{dim}(X) \geq 1$.

Proposition 3.1. Let $j$ be a nonnegative integer with $j \leq 2 d$ and $\mathbf{b}_{j}(\bar{X}) \neq 0$. Let $a$ be an integer. Assume that the Galois action on $H^{j}\left(\bar{X}, \mu_{\ell^{r}} \otimes a\right)$ is trivial. Then

$$
\bar{\chi}_{\ell^{r}}^{2 a-j}(g)=1 \forall g \in G=\mathrm{G}_{K} .
$$

Proof. Let us put $U:=H^{j}\left(\bar{X}, \mathbb{Z}_{\ell}(a)\right)$ : it is provided with the natural structure of $G=\mathrm{G}_{K}$-module. Then the universal coefficients theorem [6. Ch. V, Sect. 1, Lemma 1.11] gives us a canonical $\mathrm{G}_{K^{-}}$-equivariant embedding

$$
U / \ell^{r} U=H^{j}\left(\bar{X}, \mathbb{Z}_{\ell}(a)\right) / \ell^{r} H^{j}\left(\bar{X}, \mathbb{Z}_{\ell}(a)\right) \hookrightarrow H^{j}\left(\bar{X}, \mu_{n}{ }^{\otimes a}\right) .
$$

Since the Galois action on $H^{j}\left(\bar{X}, \mu_{n}{ }^{\otimes a}\right)$ is trivial, it is also trivial on $U / \ell^{r} U$. We have (in the notation of Sect. 2.1)

$$
U_{E}=H^{j}\left(\bar{X}, \mathbb{Z}_{\ell}(a)\right) \otimes_{\mathbb{Z}_{\ell}} \mathbb{Q}_{\ell}=H^{j}\left(\bar{X}, \mathbb{Q}_{\ell}(a)\right) .
$$

Let $V:=H^{2 d-j}\left(\bar{X}, \mathbb{Z}_{\ell}(d-a)\right)$ : it has the natural structure of $G=\mathrm{G}_{K}$-module and

$$
V_{E}=H^{2 d-j}\left(\bar{X}, \mathbb{Z}_{\ell}(d-a)\right) \otimes_{\mathbb{Z}_{\ell}} \mathbb{Q}_{\ell}=H^{2 d-j}\left(\bar{X}, \mathbb{Q}_{\ell}(d-a)\right) .
$$

The cup product pairing gives rise to a $\mathbb{Z}_{\ell}$-bilinear $\mathrm{G}_{K}$-invariant pairing known as Poincaré duality (6, Ch. VI, Sect. 11, Cor. 11.2 on p. 276], 4, p. 23], [3, Ch. II, Sect. 1])

$$
e: H^{j}\left(\bar{X}, \mathbb{Z}_{\ell}(a)\right) \times H^{2 d-j}\left(\bar{X}, \mathbb{Z}_{\ell}(d-a)\right) \rightarrow \rightarrow H^{2 d}\left(\bar{X}, \mathbb{Z}_{\ell}(d)\right) \cong \mathbb{Z}_{\ell} .
$$


It is known [10] that the induced pairing of free $\mathbb{Z}_{\ell}$-modules of finite rank

$$
e: H^{j}\left(\bar{X}, \mathbb{Z}_{\ell}(a)\right) / \text { tors } \times H^{2 d-j}\left(\bar{X}, \mathbb{Z}_{\ell}(d-a)\right) / \text { tors } \rightarrow \mathbb{Z}_{\ell}
$$

is perfect and unimodular.

Let us choose an invertible very ample sheaf $\mathcal{L}$ on $X$ and let

$$
h \in H^{2}\left(\bar{X}, \mathbb{Q}_{\ell}(1)\right)^{\mathrm{G}_{K}} \subset H^{2}\left(\bar{X}, \mathbb{Q}_{\ell}(1)\right)
$$

be its first $\ell$-adic Chern class. If $j \leq d$ then the Hard Lefschetz Theorem ([2, 3, Ch. IV, Sect. 5, pp. 274-275]) tells us that cup multiplication by $(d-j)$ th power

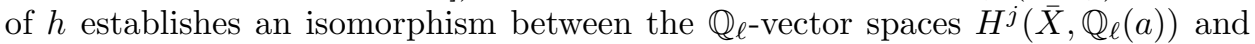
$H^{2 d-j}\left(\bar{X}, \mathbb{Q}_{\ell}(a+d-j)\right)$. On the other hand, if $d \geq j$ then cup multiplication by the $(j-d)$ th power of $h$ establishes an isomorphism between $\mathbb{Q}_{\ell}$-vector spaces $H^{2 d-j}\left(\bar{X}, \mathbb{Q}_{\ell}(a+d-j)\right)$ and $H^{j}\left(\bar{X}, \mathbb{Q}_{\ell}(a)\right)$. In both cases the Galois-invariance of $h$ implies that the $\mathbb{Q}_{\ell}$-vector spaces $U_{E}=H^{j}\left(\bar{X}, \mathbb{Q}_{\ell}(a)\right)$ and $H^{2 d-j}\left(\bar{X}, \mathbb{Q}_{\ell}(a+d-j)\right)$ are isomorphic as $\mathrm{G}_{K}$-modules. On the other hand, the $\mathrm{G}_{K}$-module

$$
\begin{gathered}
H^{2 d-j}\left(\bar{X}, \mathbb{Q}_{\ell}(a+d-j)\right)=H^{2 d-j}\left(\bar{X}, \mathbb{Q}_{\ell}(d-a+2 a-j)\right)= \\
H^{2 d-j}\left(\bar{X}, \mathbb{Q}_{\ell}(d-a)\right) \otimes_{\mathbb{Q}_{\ell}} \mathbb{Q}_{\ell}(2 a-j) \cong V_{E}(\chi)
\end{gathered}
$$

where

$$
\chi:=\chi_{\ell}^{2 a-j}: G=\mathrm{G}_{K} \rightarrow \mathbb{Z}_{\ell}^{*}=\Lambda^{*} .
$$

So the $G$-module $U_{E}$ is isomorphic to $V_{E}(\chi)$ and Theorem 2.4 tells us that

$$
\bar{\chi}_{\ell^{r}}^{2 a-j}(g)=\left(\chi_{\ell}(g)\right)^{2 a-j} \bmod \ell^{r} \mathbb{Z}_{\ell}=\chi(g) \bmod \ell^{r} \mathbb{Z}_{\ell}=1 \forall g \in G=\mathrm{G}_{K} .
$$

Proof of Theorem 1.8. Since $\mathbf{b}_{j}(\bar{X}) \neq 0$, we have $j \leq 2 d$. Recall that $n$ is a positive integer that is not divisible by $\operatorname{char}(K)$. Let $\ell$ be a prime dividing $n$ and let $\ell^{r_{n}(\ell)}$ be the exact power of $\ell$ that divides $n$. Applying Proposition 3.1 to all such $\ell$ with $r=r_{n}(\ell)$ and using Remarks 1.2 and 1.5, we obtain that the character $\bar{\chi}_{n}^{2 a-j}$ is trivial, which gives as the first assertion of Theorem 1.8. On the other hand, we know that $\bar{\chi}_{n}^{\phi(n)}$ is trivial. This implies that if $2 a-j$ and $\phi(n)$ are relatively prime then $\bar{\chi}_{n}$ is itself trivial, i.e., $\mu_{n} \subset K$. This proves the second assertion of Theorem 1.8 .

Now we use Theorem 1.8 in order to prove Theorems 1.9 and 1.10

Remark 3.2. In the statement of Theorem 1.8 we do not require that $j$ is odd and therefore its immediate Corollary 1.9 remains true without this assumption. However, if we drop this assumption in Corollary 1.9 (while keeping all the other ones) and assume instead that $j$ is even then $2 a-j$ is also even and therefore $\phi(n)$ is odd, because it is relatively prime to $2 a-j$. This implies that $n=2$ and therefore $\operatorname{char}(K) \neq 2$ and $K$ does not contain a primitive square root of unity, i.e., $K$ does not contain -1 , which is absurd.

Remark 3.3. The second assertion of Theorem 1.8 (and its proof) remains true (valid) if in its statement we replace $\phi(n)$ by its divisor $\exp (n, K)$.

Proof of Theorem 1.6. Since $a=(j \pm 1) / 2$, the integer $2 a-j= \pm 1$ is relatively prime to $\phi(n)$. Now the result follows from already proven Theorem 1.8 , 
Proof of Theorem 1.10. Suppose that the Galois action on $H^{j}\left(\bar{X}, \mu_{n}{ }^{\otimes a}\right)$ is trivial for some absolutely irreducible smooth projective variety $X$ with $\mathbf{b}_{j}(\bar{X}) \neq 0$. By Theorem 1.8, the character $\bar{\chi}_{n}^{2 a-j}$ is trivial. On the other hand, since $f:=2 a-j$ is odd and $\left[K\left(\mu_{n}\right): K\right]$ is even, Remark 1.1 tells us that $\bar{\chi}_{n}^{2 a-j}$ is nontrivial. This gives us a desired contradiction.

\section{REFERENCES}

[1] P. Deligne, La conjecture de Weil. I. Publ. Math. IHES 43 (1974), 273-307.

[2] P. Deligne, La conjecture de Weil. II. Publ. Math. IHES 52 (1980), 137-252.

[3] E. Freitag, R. Kiehl, Etale cohomology and the Weil conjecture. Ergebnisse der Math. 3 Folge, Band 13, Springer Verlag, Berlin Heidelberg New York, 1988.

[4] N.M. Katz, Review of $l$-adic cohomology. In: Motives. Proc. Symp. Pure Math. 55, Part 1. American Mathematical Society, Providence, RI, 1994, 21-30.

[5] S. Lang, Abelian varieties, 2nd edition. Springer-Verlag, New York, 1983.

[6] J.S. Milne, Étale Cohomology. Princeton University Press, Princeton, NJ, 1980.

[7] J.-P. Serre, Lectures on Mordell-Weil Theorem, 2nd edition. Aspects of Mathematics E6, Friedr. Viehweg \& Son, Braunschweig, 1990.

[8] Yu. G. Zarhin, The Brauer group of an abelian variety over a finite field. Izv. Akad. Nauk SSSR Ser. Mat. 46 (1982), 211-243; Mathematics of the USSR-Izvestiya 20 (1983), 203-234.

[9] Yu. G. Zarhin, Torsion of abelian varieties, Weil classes and cyclotomic extensions. Math. Proc. Camb. Phil. Soc. 126 (1999), 1-15.

[10] Yu. G. Zarhin, Poincaré duality and unimodularity. In: Geometry and Arithmetic, van der Geer Festschrift (C. Faber, G. Farkas, R. de Jong, eds.), pp. 369-376. European Mathematical Society, Zürich, 2012.

Department of Mathematics, Pennsylvania State University, University Park, PA 16802, USA

E-mail address: zarhin@math.psu.edu 